\section{A COURSE OF}

\section{LECTURES ON SURGERY,}

DRLIVEREE IN THE

MEDICAL SCHOOL OF CAMBRIDGE.

By GEORGE MURRAY HUMPHRY, Esq., Downing College, Surgeon to Addenbrooke's Hospital.

\section{Lecture XXV.}

Hypertrophy Continued-Tumours.

Tumours are examples of circumscribed hypertrophy no less than the growths described in the preceding lectures;-Reasons for thinking that they are so, and that they are not strictly new formations; each species of simple tumour is the representative of one of the lower orders of tissues, viz., the fatty, the fibrous, the cartilaginous, and the osseous; each originates in its corresponding tissue, at the parts where that tissue is most abundant; the structure of the tumour is often continuous with that of the tissue in which it lies: the latter is sometimes involved to a considerable extent;-analogy of tumours to warts and polypi; ihe excessive growth on which a tumour depends is attended also with a change in the develop. mental properties of the tissue concerned, leading to variations of structure; subject illustrated by some peculiarities of foetal tumours and tumours in animals;-changes taking place in tumours from progressive development, from degeneration of structure following the cessation of growth, from decay, from inflammation; tumours may be sometimes new formations; assistances to the diagnosis of tumours derived from a knowledge of the above facts; practical remarks.

WHEN we commenced the subject of hypertrophy I told you that this term would be found, without any forced interpretation of its meaning, to include a great variety of morbid products which had hitherto received no definite position-no position, that is to say, at all explanatory of their real nature-in any classification of pathology. The truth of this remark has, I think, already been sufficiently proved. The word hypertrophy in its most literal signification, imports excessive 'nutrition, in other words, nutrition beyond the natural requirements; and the effects of such excess are evinced either in the preternatural bulk, or in the preternatural structural development of the part affected, or in both of these taking place together. It has been the common practice among pathologists to confine the use of the term hypertrophy to those manifestations of its influence which are observed in whole tissues or structures. The preternatural enlargement of a whole heart, a whole liver, or a whole limb, has gone by the name of hypertrophy. But there seems no good reason for this limitation of the term, no reason against including under the same term those circumscribed enlargements and excessive developments of structure, which have been described in the two or three last lectures. Indeed, unless we use the term to express a physiological rather than a pathological condition, these products have a claim to be regarded as instances of hypertrophy in preference to those which are usually recognized as such. What, for instance, can be more truly an example of hypertrophy, in the strictest sense of the term, than a nævus, a wart, or a polypus; each of these manifestly consists in the enlargement, the excessive growth of the tissue or tissues concerned. It is true that the hypertrophy is in such cases confined to a very limited space, but it is not on that account the less well marked, the less simple in its nature, the less deserving its name. The causes which induce this circumscribed hypertrophy are much the same as those which give rise to it in a more general form; sometimes it is evidently of an inflammatory nature; sometimes a slight but continued irritation, such as the presence of dirt or moisture upon the skin, is sufficient to induce it; at others it occurs without our being able to assign any obvious reason.

These few remarks upon hypertrophy, more particularly upon hypertrophy taking place in a circumscribed manner, will serve to remind you of what has been already said respecting the nature of warts, polypi, cysts, and other formations of the like kind; they will also prepare you for what I have to say respecting the nature of the more solid growths, called " tumours," which we have now to discuss. It will be one of my objects in the present lecture to explain to you my reasons for thinking that the greater number, if not all, of these growths may be attributed to a process of circumscribed hypertrophy taking place in the tissues which form their nidus.

You all know well enough what is meant by the word " tumour." It is, therefore, unnecessary to attempt any definition. Such attempt is, indeed, better avoided; it must be unsatisfactory, because it would presuppose an acquaintance with the nature and characters of the structures in question; and would be more likely to contract your ideas than to impart information. At the present time it can scarcely be said that pathologists accredit any particular theory of their nature or origin. The views of Hunter, Abernethy, and others, that tumours originate in a coagulum of blood or a mass of lymph shed among the tissues and acquiring a power of growth \&c., are now so little regarded, that it is scarcely necessary even to allude to them. The only opinion at all commonly entertained with regard to the morbid products of this class is that they are entirely new formations, quite distinct from the original tissues, and, except that they derive their sustenance through them, in a great measure independent of them. Hunter describes a tumour to be "an entirely new-formed substance, and of new structure" - " a circumscribed enlargement in a part from disease, not strictly a disease of a natural circumscribed part." This opinion is prevalent throughout his writings, although he includes warts, polypi, and a variety of structures under the same head; and this opinion is current at the present time both in our own country and abroad. The word used by the German pathologists to signify tumours being expressive of the same view. That such an idea has been so long and so generally maintained is no 
wonder; for tumours, more particularly those of large size, do often appear to be quite separate and independent from the surrounding structures, have little sympathy with them, produce only a mechanical effect upon them, and may be removed without their loss being in any way felt by the rest of the economy. Such facts are sufficient to induce the belief that tumours are entirely distinct and new formations; but they by no means prove it, and they are quite compatible with a theory which attributes these structures to a morbid enlargement - an hypertrophy - of a circumscribed portion of the naturally existing tissues. Warts, polypi, and cysts stand in all, or nearly all these respects, in the same relation with tumours; and I think there are sufficient reasons for believing that these several morbid growths are very nearly allied in the mode of their origin as well as in the changes they undergo.

The observations made in this lecture apply almost exclusively to those which are called simple tumours. The malignant or cancerous growths we will leave till another day.

The simple tumours may be divided into four chief species, which derive their names from the appearance and more obvious characters of the structures which compose them. They are the fatty, the fibrous, the cartilaginous, and the osseous. The remark is here at once suggested, that each one of the simple or common tissues, forming the elementary basis, as it were, of the frame, has its representative species among the tumours; no structures corresponding to the higher and more gifted constituents of the frame being found in these growths. We do not meet with muscular, glandular, or nervous tissue in them; or if they do contain such tissues at all, it is in a very small proportion in comparison with the other ingredients in their composition.

Secondly. Each of these species of tumours is found to originate in its corresponding tissue. Thus the fatty tumour occurs in the adipose tissue ; the fibrous tumour occurs among fasciæ, in tendinous structures, in the uterus, and in other parts where the fibrous element predominates; the cartilaginous and osseous tumours grow in the substance and in the immediate neighbourhood of the bones. Considering the fact that each of these tissues is disseminated very generally throughout the system, and that some of them enter into the composition of nearly every organ, it is a matter of surprise that we are able to establish any such rule as this, still more that it should be a rule so little liable to exception as we shall find this to be when we come to investigate these several growths in detail. Indeed the rule may be expressed with even more stringency than $I$ heve laid it down, for it is not simply true that each of these species of tumours originates in its corresponding tissue, but it is further true that each of these species of tumours is confined, with very few exceptions, to the regions where its corresponding tissue exists in greatest abundance. Thus fatty tumours occur chiefly in the subcutaneous adipose tissue; fibrous tumours about the periosteum and in the uterus. You will search a long time without finding either a fatty, a fibrous, or a bony tumour, in a liver, a kidney, a lung, or a brain. Again, fibrous tumours are common enough in the uterus, but I never heard of a fatty tumour being developed in that organ; neither have I ever seen a fatty or a fibrous tumour growing in the interior of a bone, although both adipose and fibrous tissue exist there in small quantities. The chief exceptions to the law we are now discussing are furnished by cartilaginous tumours; these we occasionally find in parts where no cartilage exists naturally, for instance, in and about the parotid gland. Indeed the true foetal cartilage of which these tumours consist has no exact type in the body of the adult, it is but a temporary structure transitional to another state. It is not easy to explain the occurrence of these cartilaginous tumours in such anomalous positions, unless we suppose that some new powers of development as well as new energies of growth, are communicated to the affected portion of tissue. To this question we shall again return.

Thirdly. The probability afforded by these facts in favour of the theory $I$ have propounded respecting the origin of tumours, is reduced almost to a certainty by the instances, and they are not few, where a continuity of structure may be distinctly traced between the tumour and the tissue in which it is imbedded. This continuity exists sometimes in so great a degree that you cannot distinguish the one structure from the other; you cannot tell where the natural tissue ends and the tumour begins. It is observable chiefly in the adipose and fibrous tumours, but may often be noticed likewise in the osseous.

Fourthly. Although its outline may be distinct, a tumour is sometimes found to involve the parent tissue to a considerable extent, just as a wart frequently springs from a wide surface of the cutis. A fatty tumour may run under the skin for several inches, not enlarging from one centre, and growing like a ball, or sending out offsets, but communicating its own growing tendency to the adjacent adipose tissue. This at least seems to be the best mode of explaining those cases where portions of other tissue, such as the fibrous prolongations of the cutis, become inclosed in, and completely surrounded by, the tumour. What is still more to the point we now and then find a fibrous tumour springing from the whole circumference of a bone; for instance, there is in the College of Surgeons a specimen of the head of a fibula, quite surrounded by, or imbedded in, a fibrous tumour growing from, or rather a fibrous tumour of, the periosteum all around the bone. I can scarcely conceive any other mode of accounting for such a position of the tumour in relation to the bone, than by supposing that the tumour consists of a hypertrophied and morbid condition of the periosteal fibres, and that this condition has spread from point to point, and from fibre to fibre, precisely in the same way that a wart increases by a communication of the hypertrophic tendency from one papilla of the cutis to another. And, as in the case of a wart, the enlarged cuticular papillæ may be seen radiating and branching in the morbid growths, so in the tumour just mentioned, and in others of the same kind, you may discern with the naked eye the 
elongated and thickened periosteal fibres radiating into the substance of the tumour.

In the greater number of cases, it must be granted, such a connection of the tumour with the parent tissue cannot be traced; neither can we expect that it should be, except in the instances where the tumour spreads along a surface, the growing tendency being communicated from one point of the parent tissue to another. It is quite evident that the influence, whatever it be, which gives an impulse to the nutritive energies of a certain portion of tissue urging it on to morbid growth, is often exceedingly limited, affecting possibly a mere atom; and the tumour resulting from an influence so limited in its operation would of course appear to be quite isolated from its parent tissue; at any rate, would present no traceable continuity of structure with it. Scarcely indeed could we infer that any such continuity had ever existed except by the analogy of other tumours where the growing tendency has been less limited. Possibly when the tumour is very circumscribed some process may take place during its growth, whereby its continuity with the parent tissue is still further destroyed, some absorption of that tissue or degeneration of it into the common areolar structure which forms the capsule of these tumours. If any isolating process of this kind takes place under such circumstances we might in this way account for the difficulty so often experienced in tracing the continuity of glandular cysts with the ducts in which they are supposed to originate.

Sufficient reasons have, I think, now been given you to warrant the opinion, at any rate to afford great probability to the opinion, that tumours (you will remember that we speak at present of simple tumours) are not strictly new formations, as they have hitherto been considered, but that they originate in a hypertrophic condition of certain limited portions of the naturally-existing tissues. This condition, extending from point to point, may involve the parent tissue to a variable extent, after the manner of a wart, or it may be confined to the spot primarily affected, when it bears much resemblance to a polypus; the difference between a tumour and a polypus consisting in the circumstance that the former displaces the structures on all sides of it, and receives vessels in its whole circumference, instead of hanging into a cavity, and being dependent on a pedicle both for the maintenance of its place and its supply of blood. This is but an accidental difference, resulting from the peculiarity of their respective positions.

We do not pretend to search among the mysteries of nutrition for the causes of the growth of tumours; sometimes a blow, a strain, or a passing inflammation appears to give the first impulse to the disease, but in the greater number of cases we cannot learn even thus much of their early history. All that we can say of the matter is, that the ordinary restrictions as to size imposed upon the integral parts of the body, according to certain laws of development, are now set at nought; the tumour breaking through the bounds of legitimate growth goes on increasing uncontrolled by the laws presiding over the rest of the body, and regardless of the evils produced by its encroachment upon other parts. In this respect a tumour differs from those results of hypertrophy of development which occur during the fotal period, and which were described in a former lecture,-such as supernumerary limbs,- - for they invariably show themselves amenable to the general laws of development. They are always modelled according to a natural shape, though they may fail to attain it completely; and, however much they may fall short, they are never found to exceed the size of the parts to which they correspond.

When speaking to you, in the last lecture, of the nature of cysts, I said that the tissue in which they originate often receives, at the time of their formation, an impulse in its developmental no less than in its growing properties; and that it, in consequence, acquires not only an increase of size, but that it may also undergo equally remarkable changes of structure. Most of the observations then made upon this subject apply with equal force to the formation of tumours. The alteration which takes place in the origin and growth of a tumour may be simply one of bulk, the whole process consisting in a mere enlargement of a given portion of tissue. This holds true of the greater number of fatty, and of some fibrous, as well as some osseous, tumours. Commonly, however, the process is not quite so simple, the morbid enlargement being attended in most instances with some alteration of structure. Perhaps certain elements of the tissue are increased in greater proportion than others; in a fatty tumour, for instance, the areolar and fibrous element may prevail to a greater extent than in the natural adipose tissue, so as to occasion considerable difference in appearance between the two structures. In the case of the fibrous tumour of the uterus the morbid growth seems to consist of a lower order of structure than the parent tissue. The true osseous tumours are generally more dense than the bone from which they spring. Further, in the cartilaginous tumours a new character seems to be imparted to the growing element; this hardly excites our surprise when, as is usually the case, a bone is the nidus of the growth, because the growth is still in perfect keeping with the parent tissue; but it certainly does seem strange that cartilaginous masses should be developed in the midst of the testicle and the parotid gland. This apparently anomalous fact must, I suppose, be explained on the supposition that a change in the developmental qualities of the tissue concerned has accompanied its excessive growth, and has produced the remarkable structural alteration in question.

You have read, doubtless, of the various and heterogeneous compounds which are often found in fœetal tumours, such as portions of cartilage, bone, intestine, \&c. ; these are probably occasioned by the activity if the developmental forces at that period of life importing peculiar characters to any abnormal growth which may then take place. It is not quite certain that these grow ths correspond altogether with the tumours of extrauterine life; one point of difference is presented by the fact that their augmentation of size is subordinate to the laws regulating the growth of the rest of the body, 
They invariably enlarge, so far as I know, only in proportion to the growth of the child, and cease to increase when the frame has attained to its full dimensions.

An extended acquaintance with the pathology of animals, would probably show that the characters of their tumours are in a measure regulated by the predominant features of nutrition in the different species, just in the same way that the cysts in the interior of bir?'s are found to contain feathers, and those in sheep to be filled with bair, as mentioned in the last lecture. The only circumstance bearing upon this point that I am acquainted with, is the great liability to bony formations observable in the tumours of most vertebrata, which is in accordance with the activity evinced in the development of bone in these animals under inflammation and other circumstances.

In the above instances, and others of the like kind might be mentioned, the peculiarities of the tumour are inherent in it, or rather, are impressed upon it at its very commencement. There are, besides, some other peculiarities of structure dependent upon changes going on at various periods of its existence. The components of the tumour may advance to a higher, or descend to a lower, order of tissue; and they may be subject to inflammation, with any or all of its various consequences.

The progressive development of structure in tumours probably does not amount to much. For the most part they present the same, or nearly the same, characters at all periods of their existence and in all parts of their substance. In the adipose tumour this is perhaps strictly the case; the little incipient fibrous tumour also very closely resembles that which has been long growing, and which has attained to great size, provided the two specimens are selected from the same organ. There may be an increasing compactness of structure, or closer interweaving of fibres, possibly a more perfect development of the elements of those fibres as time advances; but it has not appeared to me that even this is by any means usually the case. The cartilaginous tumours afford the best examples of this sort of change, for ossifying nuclei may be often seen in the nodules which compose the mass. It seems, however, that even in these tumours ossification is less frequent than degeneration. Doubtless there must be in each tumour a regular process of development, by which its tissue is eliminated from the primary cell or the plastic fluid of the blood; and we have every reason to suppose that this takes place in the same general manner as in the natural formation of the corresponding structure in other parts of the body. But it does not appear that any material alteration takes place in tumours as the result of a tendency to progressive development, except in the instance just quoted of the conversion of cartilage into bone.

In the process of nutrition as conducted in the natural structures there is a continual mutation of particles-a never ceasing formation and disintegration of atoms. This takes place in accordance with certain inscrutable laws which we call the laws of nutrition. The rate at which it occurs is regulated by the activity of function in each particular organ; and, as a general rule, some exercise of function is essential to the proper maintenance of structure, except in the early periods of existence, when the impulse of growth operates, instead of that of function. In tumours, doubtless, the same unceasing mutation of particles is evet in progress, for it seems to be an essential attendant on nutrition under any circumstances throughout the animal and vegetable kingdoms. The continuance of that process in a perfect manner, and the proper maintenance of structure, must in these morbid masses be entirely dependent upon the stimulus of growth, for they are utterly devoid of function, and when the stimulus of growth fails, they necessarily decline or decay. Thus we generally find that when a tumour ceases to enlarge, that is to grow, it begins to show the signs of atrophy. These signs correspond with those observed in the natural tissues, consisting of wasting and degeneration of structure. As we proceed with the consideration of the several kinds of tumours I shall have frequent occasion to mention both these results. The wasting or shrinking of a tumour is by no means an uncommon event, particularly in the adipose species. The degeneration of structure is still more commonly witnessed; it most frequently takes the form of calcareous transformation, affecting chiefly the fibrous and the cartilaginous species. Here is a specimen of a fibrous tumour of the uterus, converted almost entirely into a hard, knotty, earthy. mass ; and here is a cartilaginous tumour of the pelvis, in which the original structure is almost entirely replaced by a powdery calcareous substance. In these, and other cases of the like kind, the calcification of the tumour is a tolerable sure sign, almost as certain as its wasting, that it had lost the stimulus of growth.

Another and more important change taking place in tumours, allied to that just mentioned, but differing from it in many respects, is their decay. In this, too, they show a general analogy to the naturally existing tissues, as well as to the entire body, for decay is one of the essential elements in nutrition, being the necessary preliminary to repair. Each component particle of the machine has its prescribed term of existence, its periods of growth, of maturity, and of decay. The last is as natural as the two former, and it differs from premature degeneration, just described, inasmuch as it does not result from any want of the proper stimulus to nutrition, any imperfection of function or cessation of growth but occurs in the ordinary process of nutrition, as a legitimate and necessary phase. It is not a disease but a natural phenomenon. Now, in the healthy body this change is observed only in the ultimate particles; it does not affect the entire organs, because the dissolution of one of them would lead to the destruction of the whole frame. They are, or should be, so constructed, so nicely balanced, that no one precedes the others in its decay. All are liable to that gradual degeneration of structure which is the attendant upon the waning activity of nutrition in age, but none of them can be said to decay while the body preserves its integrity. If they do so it is a premature event occasioned by 
original imperfection in their nutritive powers, or by disease, and is fatal in its consequences-is an accident rather than a natural occurrence. Each organ is a link in the chain which forms the body; the ultimate particles of those links are incessantly decaying and being reproduced, but the dissolution of a single link would be fatal to the integrity of the whole. Tumours, I have said, present a general analogy, in the mode of their nutrition and in their decay, to the other structures of the body; it is, however, only a general analugy, for they differ from the ordinary components of the body in the important particular that they do not harmonize wth them as to the prescribed term of their duration; they begin, run their course, and decay quite irrespectively of the rest of the body, sometimes long before it has completed its allotted period. In the case of simple tumours it is true the term of existence is commonly protracted, so much so indeed, that generally decay is by no means ordinarily observed in them, and this constitutes one of the grand features of distinction between simple and malignant tumours, the latter being very prone to early decay, as we shall find when we come to the description of these destructive growths.

The decay of a tumour, which you will understand is to be regarded as a different process from its degenerationthe one occuring as a regular phenomenon, the other as the result of a cessation of growth, and a consequent failure of nutrition-generally displays itself in a loosening and softening of its component parts, which may proceed to actual liquefaction, so that the whole tumour is converted into a dirty pultaceous or diffluent mass contained in the capsule which prevents its escape among the surrounding tissues. The dissolution, especially if it goes on quickly, is generally attended with some inflammatory disturbance, which causes the admixture of pus with the contents of the tumour, and may lead to their discharge by inducing the absorption or ulceration of the adjacent tissues. It generally commences at the centre of the tumour, and may be confined to one part, or may affect the whole substance about the same time. Such softened tumours, with their investing capsules, have been mistaken for cysts, and tapped as such. The fibrous and cartilgainous varieties are most frequently affected in this manner, and $I$ shall have more to say of it when speaking of those tumours in detail.

Although inflammation sometimes take place in tumours, we cannot say that it is of frequent occurrence, which is no wonder seeing that in their want of any function they are free from the chief exciting causes of that process. When it does take place its effects are similar to those observed in other tissues. It greatly facilitates softening, and may induce suppuration, ulceration, or even mortification. These results are most likely to occur in the parts of a tumour exposed to friction or some external source of irritation.

From what has been said you are not absolutely to infer that a tumour is in nocase a new formation; there are, to all appearances, some instances where it is altogether an extraneous structure superadied to the rest of the frame. Such are the masses of lymph effused upon the surface of serous membranes, and acquiring the property of growth although the inflammation which induced them may have subsided. I have several times called your attention to these lymph-masses or lymph-tumours, often found upon the peritoneal covering of the spleen and liver, and bave told you that they seem to arise, in many cases, without any preceding or accompanying inflammatory symptoms; nevertheless they resemble the ordinary products of inflammation, and appear to consist of condensed and altered lymph. There is, however, some little obscurity attached to these formations, and it is not, therefore, wise to insist too much upon any inferences drawn from them.

The points relating to the mode of origin and growth, \&c., of tumours which we have been considering, suggest a few practical remarks.

In the first place we derive great assistance in the diagnosis of simple tumours in general, and of their several varieties, by a knowledge of the fact that they are of most frequent occurrence in the regions where the simple tissues-the adipose, the fibrous, and the osseous $\rightarrow$ are most abundant; that they are, indeed, nearly confined to these regions. It is a further assistance to $\mathrm{kndw}$ that the character of the simple tumour bears for the most part a general, often indeed a close, resemblance to that of the tissue in which it grows. If, forinstance, a tumour be formed in the subcutaneous stratam, the chances are greatly in favour of its being adipose. If it be connected with a bone, it is rot likely to be fatty, but it may be fibrous, cartilaginous, or osseous. If $/$ in the uterus, it is almost sure to be fibrous. Suppose we are called upon to decide the nature of a tumour seated beneath the fascia, but not connected with the bone : we know that simple tumours do not occur in the muscles, that they are not common in the fine cellular or adipose tissue, between the muscles; our apprehensions, are, therefore, awakened, and we deal cautiously with it, fearing that the growth may be malignant. In like manner a solid tumour, formed in one'of the internal organs-in the stomach, the spleen, or the lungswe may decide with tolerable certainty not to be of simple nature. These general rules you will find of great advantage in practice, and the exceptions to which:they are liable are not sufficient to detract from their value.

The facts already mentioned,-that simple tumours generally grow slowly; that they do not necessarily exert much deleterious influence upon the constitution, or upon the neighbouring parts, independently of their pressure; that they may cease to enlarge, and even waste away-should be remembered, and should, be allowed to have their weight when you are contemplating the question of an operation for their removal. The knowledge of these circumstances will sometimes induce us to refrain from interference when the tumour is seated deeply, or when the incisions required for its extirpation would be attended with danger or a probability of injury to a joint or large vessel.

When you do set about the removal of a simple tumour it is well to know that its boundaries are not always clearly defined, and that in consequence you may find a difficulty in distiguishing the healthy from 
the morbid tissue. In such case, of course, the disease is not unlikely to return, because its extirpation may have been incomplete. This $I$ apprehend to be the reason that simple tumours have in some cases been tbought to possess malignant characters. Generally the tumour has no close connection with any surrounding tissues; it lies like a ball in the midst of them they being only stretched and compressed by it, so that it may be cut away without loss to any other structure, and is not likely to return when thus isolated.

The occurrence of several tumours in the same tissue, or in tissues of the same class, is no evidence of their being other than simple in their nature. Moreover the fact of one class of tissues being thus affected does not in the slightest degree increase the probability that any other is likely to be diseased in a similar manner. Thus the uterus is not more likely to be the seat of fibrous tumours because there are fatty growths in the subcutaneous tissue. Neither under ordinary circumstances do we find that these simple tumours are associated with any particular condition of the general health. They occur in the robust and vigorous as well as in the feeble and sickly; in the young, the middleaged, and the old, alike. There seems to be scarcely any sympathy, any evidence of mutual action and reaction, between them and the constitution. No noxious influence radiates from them, and, except for their bulk, the weight, pressure, disfigurement, \&c., which they occasion, they may be regarded as harmless. Even the progress of softening, of suppuration, and ulceration in them, is not necessarily attended with any appreciable affect upon the system. Such being the case, we are not surprised that medicinal agents intended to operate through the constitution, are productive of so little change in tumours. We hear now and then of the good results of iodine, liquor potassæ, and other medicines supposed to rouse the activity of the absorbents; but they are for the most part utterly useless, and will undermine the health long before the tumour shows any signs of yielding to their sway.

Sometimes, it is true, we observe that a simple tumour, which had for some time been indolent, and had scarcely attracted attention, acquires a new impulse in its growth and becomes a source of uneasiness when the patient's health is disordered. In such cases we, of course, regulate the treatment accordingly, in the hope that a restoration of the health will be followed by a return of the tumour to its quiescent state. More commonly it is to be remarked that the growth of a tumour is influenced by the movements of the limb or foot in which it forms. I have known two or three instances where the complete rest or more moderate exercise of the limb has been followed by a total cessation of the enlargement of the tumour. A remarkable case of the kind occurred in the hospital five or six years ago, in the person of a young woman who became a patient on account of a tumour, apparently of fibrous character, involving the head of the fibula. It had been steadily increasing up to the time of her admission, and she was willing to submit to an operation for its removal. In order to secure the complete extirpation of this mass it would have been necessary to cut away the upper end of the fibula, to divide the peroneal nerve, and do other injury to the limb. We determined, therefore, in the first instance, to try the effect of complete rest, watching the progress of the case for a time. She was accordingly kept in bed for some weeks, and there having been no evidence of any increase in the size of the tumonr during that period, she went home continuing the treatment in a modified way. No recurrence of activity in the growth has taken place from that time to this. The mass gradually became very hard, but the muscles and other structures accommodated themselves to it, and it does not interfere with the movements or usefulness of the limb in any degree. The same results have followed similar treatment in other cases, and the hint afforded by them is worth bearing in mind.

In the next lecture we will consider the effects produced by simple tumours upon the surrounding tissues, the mode of formation of their capsules, \&c.

\section{CASE OF}

\section{MALFORMED AND IMPERFORATE VAGINA.}

\section{By CHARLES MAYO, EsQ.; F.R.C.S.,} BURGEON TO THE WINCHESTER COUNTY HOSPITAL.

Read at the Eighteenth Anniversary of the Provincial Medical axd Surgical Association, held at Hull, August 7th and 8th, 1>50,

JANE INARD, aged 19, was admitted into the hospital March, 1824, under the care of the physician, for amenorrhœa, with some swelling and hardness in the lower part of the abdomen. The physician prescribed various medicines to remove the obstruction, and directed mercurial frictions on the tumefied parts, and after some time I was directed to examine the swelling. I found a hard incompressible tumour in the hypogastric region, and more particularly prominent in the left groin, so as to give one the idea of some glandular swelling propagated in the course of the internal chain of inguinal glands. I was not at that time aware that she had never menstruated, and it was not till after some days that she disclosed to the nurse that she had been examined, per vaginam, by a surgeon at Basingstoke, who had pronounced that a preternatural formation of the sexual organs was the occasion of delayed menstruation. 18th.-On examination of the genitals I found that the vagina was entirely closed, the orifice of the meatus urinarius being within a line or two of the inferior angle formed by the labia pudendi, which, with the nymphæ, were completely formed. I passed a catheter into the bladder, and having drawn off the urine, my assistant held it firm in its situation, while I endeavoured to dissect along the course of it, in the direction of the vagina upwards and inwards; for this purpose I used various directors and probe-pointed bistouries, till at length after stretching the divided parts with the point of the finger, I was able to feel the distended uterus at 